\title{
Possible Magnetic Resonance Signal Due to the Movement of Counterions around a Polyelectrolyte with Rotational Symmetry
}

\author{
José A. Fornés \\ Departamento de Fsica Aplicada I. Facultad de Ciencias Físicas, Universidad Complutense, Madrid, Spain \\ Email: jafornes@fis.ucm.es
}

Received 6 January 2015; accepted 27 January 2015; published 3 February 2015

Copyright (C) 2015 by authors and Scientific Research Publishing Inc.

This work is licensed under the Creative Commons Attribution International License (CC BY). http://creativecommons.org/licenses/by/4.0/ c) (i) Open Access

\begin{abstract}
Experimental, theoretical and computational studies revealed that the characteristic time scales involved in counterion dynamics in polyelectrolytes systems might span several orders of magnitude ranging from subnanosecond times to time scales corresponding to acoustic-like phonon mode frequencies, with an structural organization of counterions in charge density waves (CDWs). These facts raise the possibility of observing Magnetic Resonance (MR) signals due to the movement of counterions in polyelectrolytes. In case that this signal is detected in macroions or other biological systems, like micelles, vesicles, organeles, etc. with rotational symmetry, this method opens a new tool to measure with precission the counterions velocity.
\end{abstract}

\section{Keywords}

Magnetic Resonance, Polyelectrolytes, Counterions, Charge Density Waves CDW, Rotational Symmetry Macroions, Nanomedicine, Biomaterials

\section{Introduction}

Polyelectrolytes are ionizing macromolecules. An important property of polyelectrolyte molecules is the formation of electric double layers surrounding the polymer chains. Most of the biological macromolecules under physiological conditions are polyelectrolytes in solution and their biological activity depends on their physico-chemical properties. Depending on the strength of the electrostatic interactions, it has been found [1] that distinct "phases" of counterions can be formed, i.e., a "condensed" layer of mobile oppositely charged counterions [2] [3] and "diffuse" phase consisting of loosely bound counterions to the considered macroion. The last phase of collective motion of the more mobile ions can be involved in the formation of charge density waves. 
Experimental [4]-[7], theoretical [8]-[14] and computational [15]-[17] studies reveal that the characteristic time scales involved in counterion dynamics in polyelectrolyte systems may span several orders of magnitude ranging from subnanosecond times to time scales corresponding to acoustic frequencies. The counterions exhibit an acoustic-like phonon mode that suggests the existence of a correlated phase. At small length scales within the domains, counterions exhibit liquid-like correlations and dynamics, and they are organized into counterion charge density waves (CDWs) [18]. The measured speed of sound is of the order of $2000 \mathrm{~m} / \mathrm{s}$.

We believe that these CDWs exist also on the surface of polyelectrolytes with rotational symmetry generating a circular current loop, which produces a magnetic field $\boldsymbol{B}$ and the corresponding magnetic moment $\boldsymbol{\mu}$ at the center of the macroion. This magnetic moment $\boldsymbol{\mu}$ is oriented in an external magnetic field $\boldsymbol{B}_{\mathrm{o}}$ producing a magnetic resonance signal under the aplication of a certain frequency $v$.

\section{Magnetic Field on the Axis of a Circular Current Loop}

Consider a circular loop of wire of radius $R$ located in the $x y$ plane and carrying a steady current $I$, as shown in Figure 1. The magnetic field at an axial point $\mathrm{P}$ a distance $z$ from the center of the loop is given by [19]:

$$
\boldsymbol{B}_{z}=\frac{\mu_{0} \boldsymbol{\mu}_{z}}{2 \pi\left(z^{2}+R^{2}\right)^{3 / 2}}
$$

where $\mu_{\mathrm{o}}$ is the permeability of free space, $\mu_{\mathrm{o}}=4 \pi \times 10^{-7} \mathrm{~Wb} \cdot \mathrm{A}^{-1} \cdot \mathrm{m}^{-1}$ and $\mu_{z}=I\left(\pi R^{2}\right) z_{0}$ is the magnetic moment associated with the current loop, where $z_{0}$ is a unit vector in the $z$ direction. The magnetic field at the center of the loop, we set $z=0$ in Equation (1). At this special point, this gives ${ }^{1}$,

$$
\boldsymbol{B}_{z}=\frac{\mu_{\mathrm{o}} \boldsymbol{\mu}_{z}}{2 \pi R^{3}} \quad(\text { at } z=0)
$$

In case that the current loop is produced by charged particles in movement, with electric charge $q$ and velocity $v$. The magnetic moment is given by,

$$
\boldsymbol{\mu}_{z}=I\left(\pi R^{2}\right) z_{\mathrm{o}}=\frac{q v R}{2} z_{\mathrm{o}}
$$

where we have used $I=\frac{q}{\tau}=\frac{q \omega}{2 \pi}$, with $\omega=\frac{v}{R}$, with $\tau$ the period and $\omega, v$ the angular and linear velocity of the counterions respectively.

\section{Magnetic Resonance}

A magnetic moment in a external magnetic field $\boldsymbol{B}_{\mathrm{o}}$ adquires an energy $E$ given by the following escalar

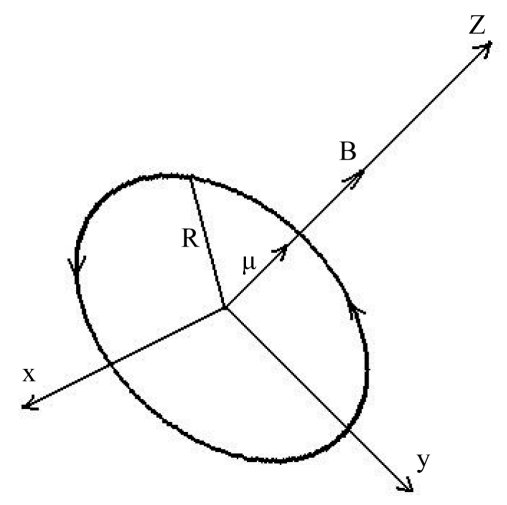

Figure 1. Magnetic field on the axis of a circular current loop.

${ }^{1}$ The SI unit of magnetic field is the tesla $(\mathrm{T}):[B]=\mathrm{T}=\frac{\mathrm{Wb}}{\mathrm{m}^{2}}=\frac{\mathrm{N}}{\mathrm{C} \cdot \mathrm{m} / \mathrm{s}}=\frac{\mathrm{N}}{\mathrm{A} \cdot \mathrm{m}}$. In practise, the cgs unit for magnetic field, called the gauss (G) $1 \mathrm{~T}=10^{4} \mathrm{G}$. 
product [20],

$$
E=-\boldsymbol{\mu}_{z} \cdot \boldsymbol{B}_{\mathrm{o}}=-\mu_{z} \cdot B_{\mathrm{o}} \cos (\theta)
$$

We observe that the highest and lowest energy is when $\theta=\pi$ and $\theta=0$, this means that when $\boldsymbol{\mu}_{z}$ and $\boldsymbol{B}_{z}$ are in opossite directions the energy is maximum and in the same directions is minimum. This last state is the most natural tendency of the magnetic dipole, paralell to the field. The difference in energy between this two states is given,

$$
\Delta E=2 \mu_{z} \cdot B_{\mathrm{o}}
$$

Reemplacing $\mu_{z}$ given by Equation (3) in Equation (5), considering $q=z e_{0}, z$ being the valence of the ions and $e_{\mathrm{o}}=1.6 \times 10^{-19} \mathrm{C}$, the elementary charge, we obtain,

$$
\Delta E=z e_{\mathrm{o}} v R B_{\mathrm{o}}
$$

As an example consider the circular movement of counterions on the surface of a polyelectrolyte, used in nanomedicine with rotational symmetry, as shown in Figure 2, this does not mean that this effect can be visualize necessarily in this molecule. These ions with charge $q$ and velocity $v$ produce a magnetic field $\boldsymbol{B}$ and a magnetic moment $\boldsymbol{\mu}$ perpendicular to the plane of the macroion in accordance to Equation (2) and Equation (3). If we put this magnetic moment in an external magnetic field $\boldsymbol{B}_{0}$, its orientation will no longer be random. The small magnetic moment may spontaneously "flip" from the most favorable orientation, the lowenergy state and the less favorable orientation the high-energy state and visceversa. The energy required to induce flipping and obtain a Magnetic Resonance (MR) signal, given by Equation (6), is shown in Figure 3 to depend on the strength of the magnetic field $B_{0}$ in which the macroion containing the magnetic moment is placed. The input radiation energy in order to accomplish the transition given by Equation (6) is given by Planck's law

$$
\Delta E=h v
$$

Using Equation (6), we obtain for the resonance frequency,

$$
v_{\mathrm{o}}=\frac{z e_{\mathrm{o}} v R B_{\mathrm{o}}}{h}
$$

where $h$ is Planck's constant $\left(6.63 \times 10^{-34} \mathrm{~J} \cdot \mathrm{sec}\right)$.

\section{Conclusions}

The distribution of molecular magnets in the different energy states is given by the Boltzmann equation,

$$
\frac{N_{\text {upper }}}{N_{\text {lower }}}=\exp \left(-\frac{\Delta E}{k T}\right)=\exp \left(-\frac{z e_{\mathrm{o}} v R B_{\mathrm{o}}}{k T}\right)
$$

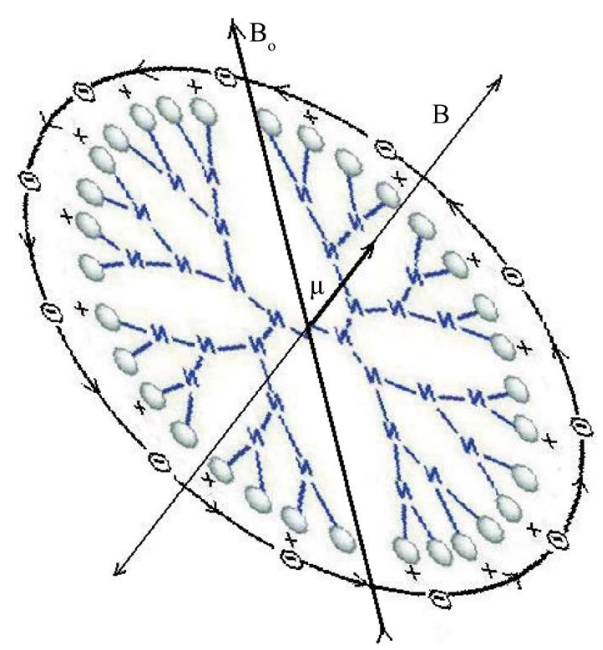

Figure 2. G3 PAMAM dendrimer surrounded by counterions with velocity $V$. 
where $N_{\text {upper }}$ and $N_{\text {lower }}$ represent the population of molecular magnetic moments in upper and lower energy states, respectively. $k=1.38 \times 10^{-23} \mathrm{~J} \cdot \mathrm{K}^{-1}$ is Boltzmann constant and $\mathrm{T}$ is the absolute temperature $(\mathrm{K})$. To give some idea of the consequences of increasing magnetic field on the population of molecular magnets states, the distribution of a small number (about two million, non real ${ }^{2}$ ) of macroions magnets, calculated from Equation (9), is shown in Figure 3.

Such a small population difference presents a significant sensitivity problem for MR because only the difference in population is detected; the others effectively cancel one another. As seen from Equation (8) and Equation (9), the use of stronger magnetic fields will increase the population ratio, and consequently the sensitivity. In Table 1, we observe that the resonance energy, varies between $(1.55-15.48) \times 10^{-3} \mathrm{kT}$ units and the resonance frequency between $(9.67-96.66) \mathrm{GHz}$ while the magnetic field varies between $(1-10) \mathrm{T}$, for a polyelectrolyte $10 \mathrm{~nm}$ radius with $q=2 e_{\mathrm{o}}$ and $V=2000 \mathrm{~m} / \mathrm{s}$.

In Table 2 are reported NMR energy and frequency data of 3 nuclei. Compared with Table 1 for the counterions, we observe that the NMR values are one to three digits lower.

In case that this signal is detected in macroions or other biological systems, like micelles, vesicles, organeles, etc. with rotational symmetry, this method opens a new tool to measure with precission the counterions velocity.

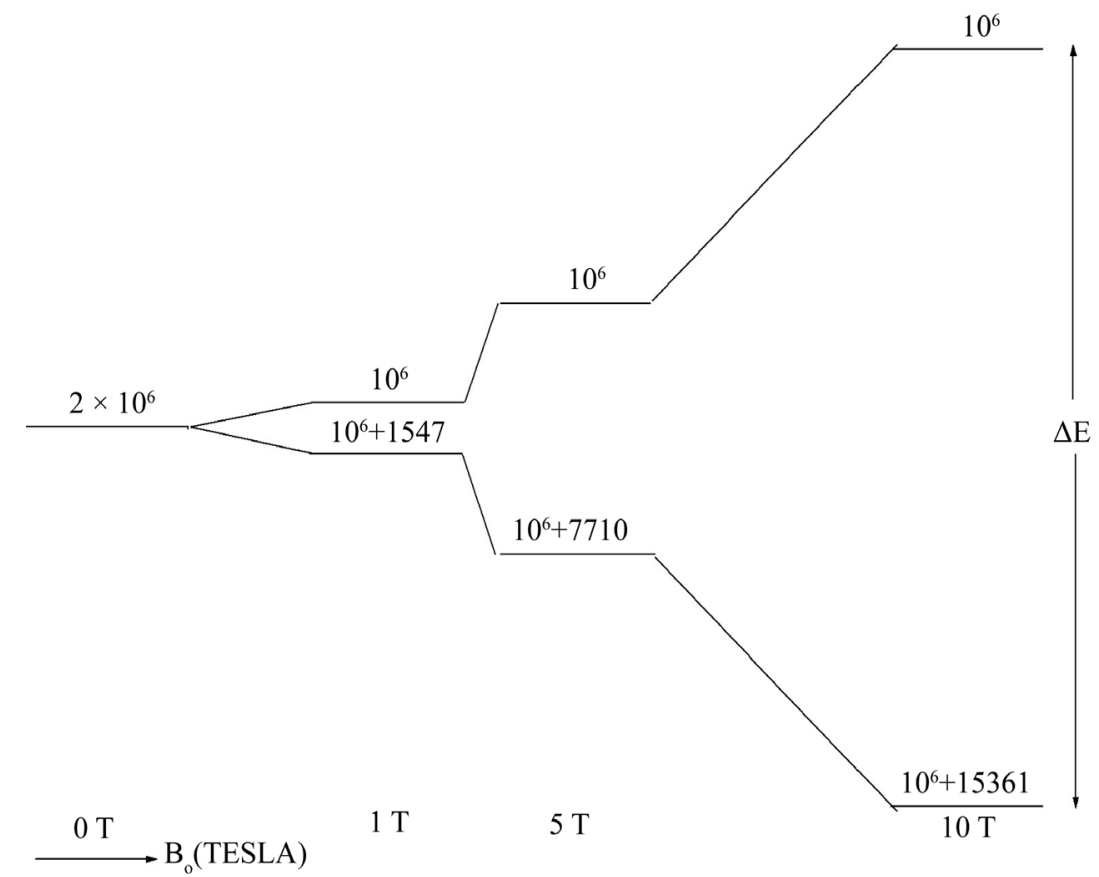

Figure 3. Dependence on magnetic field strength $B_{o}$ of $\Delta E$ and the relative populations of the energy levels for counterions with $z=2$ and velocity $V=2000 \mathrm{~m} / \mathrm{s}$ and a macroion with $R=10 \mathrm{~nm}$.

Table 1. Results shown in Figure 3.

\begin{tabular}{ccccc}
\hline$B_{o}(\mathrm{~T})$ & $\mu_{\uparrow}$ & $\mu_{\downarrow}$ & $\frac{\Delta E}{k T} 10^{-3}$ & $\nu_{\circ}(\mathrm{GHz})$ \\
\hline 0 & $2 \times 10^{6}$ & $2 \times 10^{6}$ & 0 & 0 \\
1 & $1 \times 10^{6}$ & $10^{6}+1547$ & 1.548 & 9.666 \\
5 & $1 \times 10^{6}$ & $10^{6}+7710$ & 7.740 & 48.331 \\
10 & $1 \times 10^{6}$ & $10^{6}+15,480$ & 15.480 & 96.662 \\
\hline
\end{tabular}

${ }^{2}$ In practice we put a huge number (approaching Avogadro's number) of molecular magnets in the sample that is placed in a magnetic field. 
Table 2. NMR for 14.1 T.

\begin{tabular}{ccc}
\hline Nuclei & $\frac{\Delta E}{k T} 10^{-5}$ & $v_{\text {。 }}(\mathrm{MHz})$ \\
\hline${ }^{1} \mathrm{H}$ & 10.00 & 600 \\
${ }^{15} \mathrm{~N}$ & 1.00 & 60.8 \\
${ }^{13} \mathrm{C}$ & 2.41 & 151 \\
\hline
\end{tabular}

The magnetic moment for phonons counterions with velocities of the order of $2500 \mathrm{~m} / \mathrm{s}$ and for a macroion of $10 \mathrm{~nm}$ radius with $q=2 e_{\mathrm{o}}$ is $\mu=4 \times 10^{-24} \mathrm{~J} \cdot \mathrm{T}^{-1}$, of the order of Bohr magneton $\mu_{B}=9.27 \times 10^{-24} \mathrm{~J} \cdot \mathrm{T}^{-1}$.

\section{References}

[1] Katsumoto, Y., Omori, S., Yamamoto, D., Yasuda, A. and Asami, K. (2007) Dielectric Dispersion of Short Single-Stranded DNA in Aqueous Solutions with and without Added Salt. Physical Review E, 75, Article ID: 011911. http://dx.doi.org/10.1103/PhysRevE.75.011911

[2] Manning, G.S. (1993) A Condensed Counterion Theory for Polarization of Polyelectrolyte Solutions in High Fields. The Journal of Chemical Physics, 99, 477-485. http://dx.doi.org/10.1063/1.465772

[3] Borukhov, I., Lee, K.C., Bruinsma, R.F., Gelbart, W.M., Liu, A.J. and Stevens, M.J. (2002) Association of Two Semiflexible Polyelectrolytes by Interchain Linkers: Theory and Simulations. The Journal of Chemical Physics, 117, 462-480. http://dx.doi.org/10.1063/1.1481382

[4] Hinderberger, D., Spiess, H.W. and Jeschke, G. (2004) Dynamics, Site Binding, and Distribution of Counterions in Polyelectrolyte Solutions Studied by Electron Paramagnetic Resonance Spectroscopy. The Journal of Physical Chemistry B, 108, 3698-3704. http://dx.doi.org/10.1021/jp036043u

[5] Prabhu, V.M., Amis, E.J., Bossev, D.P. and Rosov, N. (2004) Counterion Associative Behavior with Flexible Polyelectrolytes. The Journal of Chemical Physics, 121, 4424-4429. http://dx.doi.org/10.1063/1.1776556.

[6] Morfin, I., Horkay, F., Basser, P.J., Bley, F., Hecht, A.M., Rochas, C. and Geissler, E. (2004) Adsorption of Divalent Cations on DNA. Biophysical Journal, 87, 2897-2904. http://dx.doi.org/10.1529/biophysj.104.045542

[7] Popov, A. and Hoagland, D.A. (2004) Electrophoretic Evidence for a New Type of Counterion Condensation. Journal of Polymer Science Part B: Polymer Physics, 42, 3616-3627. http://dx.doi.org/10.1002/polb.20200.

[8] Schwarz, G. (1956) Zur Theorie der Leitfähigkeitsanisotropie von Polyelektrolyten in Lösung. Zeitschrift für Physik, 145, 563-584.

[9] Schwarz, G. (1959) Über die Dispersion des Orientierungsfeldeffektes von Polyelektrolyten in hoehfrequenten elektrischen Feldern. Zeitschrift für Physikalische Chemie, 19, 286-314. http://dx.doi.org/10.1524/zpch.1959.19.5_6.286

[10] Mandel, M. (1961) The Electric Polarization of Rod-Like, Charged Macromolecules. Molecular Physics, 4, 489-496. http://dx.doi.org/10.1080/00268976100100651

[11] Fornés, J.A. (1998) Thermal Electrical Fluctuations around a Charged Colloidal Cylinder in an Electrolyte. Physical Review E, 57, 2104-2109. http://dx.doi.org/10.1103/PhysRevE.57.2104

[12] Fornés, J.A. (1998) Fluctuation-Dissipation Theorem and the Polarizability of Rodlike Polyelectrolytes: An Electric Circuit View. Physical Review E, 57, 2110-2114. http://dx.doi.org/10.1103/PhysRevE.57.2110

[13] Fornés, J.A. (2000) Dielectric Relaxation around a Charged Colloidal Cylinder in an Electrolyte. Journal of Colloid and Interface Science, 222, 97-102.

[14] Kim, W.K. and Sung, W. (2008) Charge Density Coordination and Dynamics in a Rodlike Polyelectrolyte. Physical Review E, 78, Article ID: 021904. http://dx.doi.org/10.1103/PhysRevE.78.021904

[15] Karatasos, K. and Krystallis, M. (2009) Dynamics of Counterions in Dendrimer Polyelectrolyte Solutions. The Journal of Chemical Physics, 130, Article ID: 114903. http://dx.doi.org/10.1063/1.3088849

[16] Lo, T.S., Khusid, B. and Koplik, J. (2008) Dynamical Clustering of Counterions on Flexible Polyelectrolytes. Physical Review Letters, 100, Article ID: 128301. http://dx.doi.org/10.1103/PhysRevLett.100.128301

[17] Chang, R. and Yethiraj, A. (2002) Brownian Dynamics Simulations of Salt-Free Polyelectrolyte Solutions. The Journal of Chemical Physics, 116, 5284. http://dx.doi.org/10.1063/1.1453396

[18] Woolf, N.J., Priel, A. and Tuszynski, J.A. (2009) Structural and Functional Roles of the Neuronal Cytoskeleton in Health and Disease. Springer, Heidelber, Dordrecht, London, New York, 88. 
[19] Serway, R.A. (1992) Physics for Scientists \& Engineers with Modern Physics. 3rd Edition, Saunders Golden Sunburst Series, Philadelphia, London, 839.

[20] Jackson, J.D. (1963) Classical Electrodynamics. 3rd Edition, John Wiley \& Sons, Inc., New York, London, 150. 
Scientific Research Publishing (SCIRP) is one of the largest Open Access journal publishers. It is currently publishing more than 200 open access, online, peer-reviewed journals covering a wide range of academic disciplines. SCIRP serves the worldwide academic communities and contributes to the progress and application of science with its publication.

Other selected journals from SCIRP are listed as below. Submit your manuscript to us via either submit@scirp.org or Online Submission Portal.
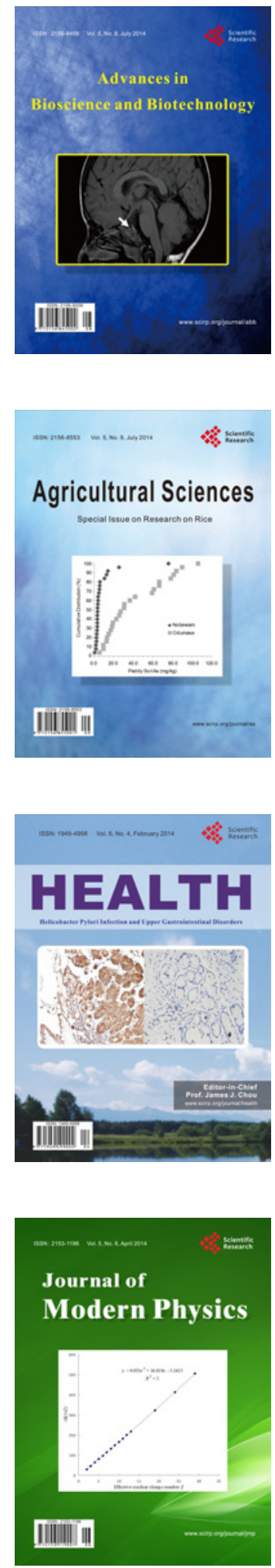
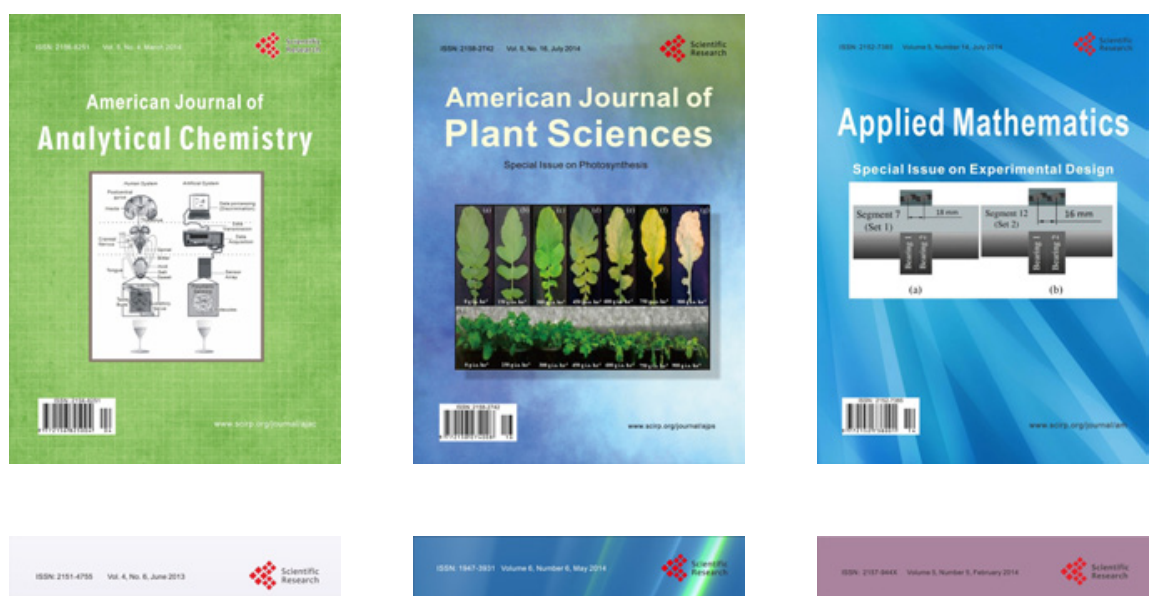

Creative Education
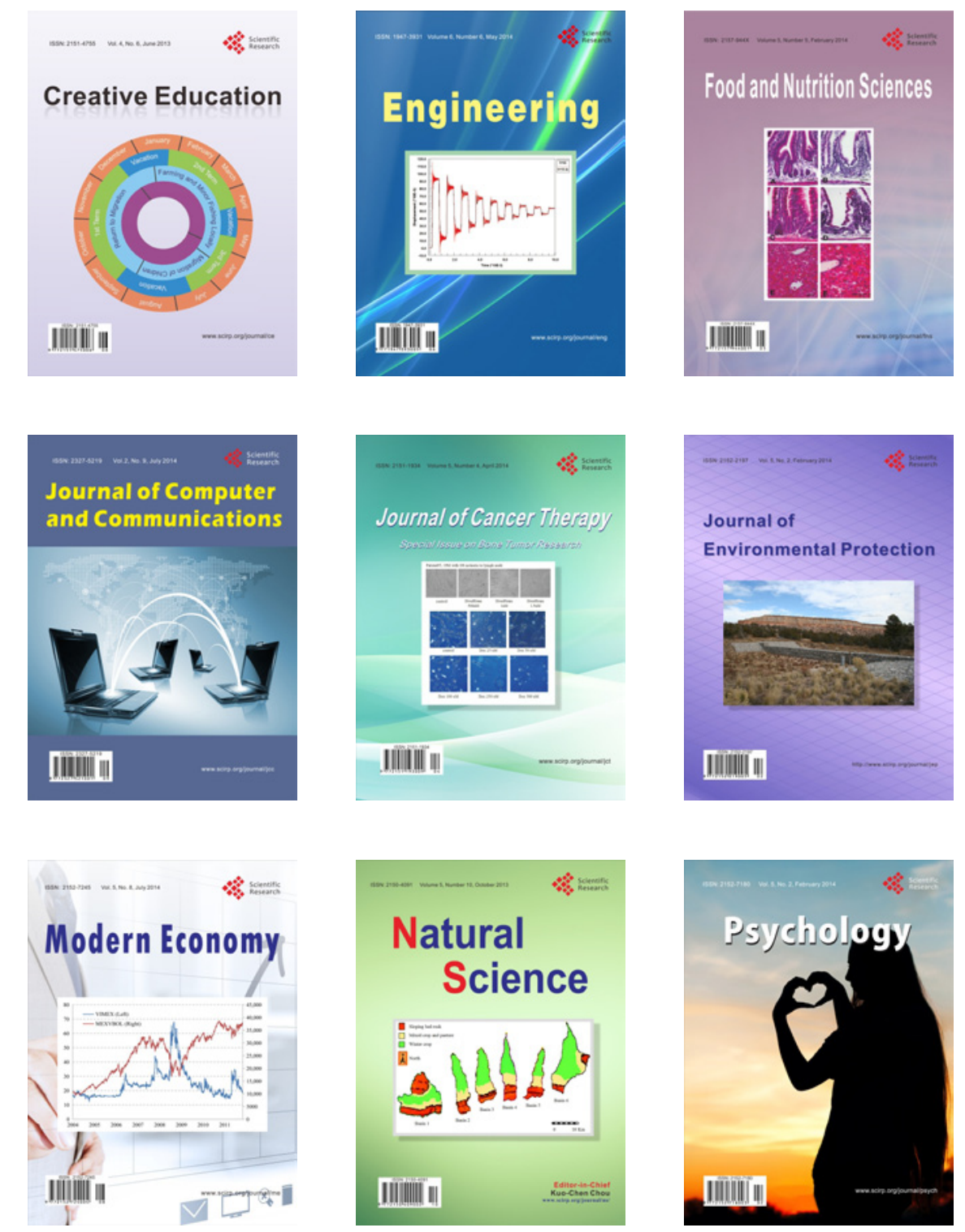